\title{
Las Revistas de Comunicación en el Panorama Internacional Análisis de la Presencia en los Sistemas de Indexación https://doi.org/10.21814/uminho.ed.43.7
}

\section{Antonio Castillo Esparcia, Ana Almansa Martínez y Elizabet Castillero Ostio \\ Departamento de Comunicación Audiovisual y Publicidad, Facultad de Ciencias de la Comunicación, Universidad de Málaga, España}





\section{Breve histórico}

Hasta hace pocos años, los investigadores en Comunicación expresaban sus análisis en diferentes ámbitos con la preponderancia alta en libros y capítulos de libros. En este sentido, la cultura de la publicación, en revistas científicas, era un elemento marginal de la estrategia de difusión de sus conocimientos.

Los inicios conceptuales de la revista como texto reducido de explicación de los resultados de una investigación y que desea ser difundido a una determinada comunidad científica, se piensa que tuvo lugar cuando las principales academias científicas del siglo XVII adoptaron un sistema de comunicación entre pares, es decir, específico para los científicos. Así, se estableció un correo de confianza a semejanza del sistema que ya utilizaban los diversos países europeos en sus comunicaciones diplomáticas u oficiales (Castillo-Esparcia \& Carretón, 2010, p. 294).

Por ello, siempre han existido mecanismos de información para conocer qué, quién, cómo y sobre qué se está investigando. En determinadas épocas los sistemas de comunicación humanos sólo permitían la creación de centros de estudios que reunían a los investigadores y que el conocimiento y las investigaciones se producían a través de las consultas personales o documentales en esos centros.

Con la irrupción de la imprenta, se pudo realizar una creciente difusión de la investigación científica y el consecuente conocimiento. En ese proceso, comenzaron a aparecer las revistas como instrumentos de difusión de las investigaciones. Y cuando las revistas aumentan en número se comienza a establecer la necesidad de que se valore para qué sirven, a quién llega y qué repercusión tienen los artículos en la comunidad científica.

Las primeras revistas científicas surgen, en el siglo XVII, como respuesta a la necesidad de intercambio de conocimientos en el ámbito científico. Así, las dos primeras revistas (Le Journal des Savants y Philosophical Transactions) surgen en 1665 (CastiLlo-Esparcia, 2011, p. 137).

Con la generalización de las revistas como instrumentos de la difusión de la investigación se aumentó el interés científico sobre su papel en la ciencia a través de análisis sobre la producción de textos o las autorías. La bibliometría se ha insertado plenamente en el quehacer de los investigadores en Comunicación y desempeña un apartado que afecta a diversos ámbitos de las publicaciones científicas (Castillo Esparcia \& Carretón, 2010):

los estudios bibliométricos permiten conocer los ámbitos en los que se desarrollan las temáticas de un campo científico, conocer las tendencias de las investigaciones, identificar a los grupos de investigación (investigadores) 
que están trabajando, verificar el grado de interconexiones internacionales entre investigaciones, la relación entre género, entre investigadores, los sistemas de citas de otras publicaciones, las autocitas de las publicaciones, las autocitas de los investigadores, los centros de investigación en los que se realizan (educativos, profesionales, investigadores). Es decir, todo un conjunto de parámetros que permiten establecer un panorama del estudio de la investigación en un campo concreto. (p. 293)

A partir de ese eje central de las revistas científicas como elementos de transmisión del conocimiento científico se han derivado toda una serie de consecuencias como son los indicadores bibliométricos para conocer el estado de la investigación de países e investigadores. Cuando hablamos de indicadores de evaluación de la investigación nos encontramos con unos criterios esencialmente cuantitativos a través de los cuales se establecen decisiones político-científicas. Así, estos indicadores se aplican en:

- $\quad$ evaluación de la producción científica de países, instituciones e investigadores. Los procesos de evaluación se concretan en la producción de textos, en la frecuencia y en los impactos de esa producción;

- $\quad$ en el caso de los países, la política científica puede condicionarla producción científica y sus modos de expresión. Así, observamos cómo el tipo de evaluación de la actividad científica condiciona las actitudes y los comportamientos de los investigadores en el momento de publicar sus investigaciones en determinadas revistas a partir de la calidad de esas revistas. Es el caso de los sistemas nacionales de investigación que inciden en la necesidad de publicar en revistas incluidas en determinadas bases de datos y de sistemas de calidad. Así, cuando las agencias de calidad científica establecen qué sistemas de indexación de las revistas son los más prestigiosos están comenzando con la determinación de en qué revistas publicar para valorar la actividad científica de los investigadores. En este ámbito, los dos principales sistemas son el Journal Citations Reports (JCR) y el Scimago Journal Ranking (SJR) (Castillo-Esparcia, 2011).

En el campo de la Comunicación, el sistema de transmisión tradicional han sido las publicaciones y dentro de ellas, los libros. La mayoría de las fuentes utilizadas en la práctica docente y en las citas de investigaciones provenían de libros. Sin embargo, los sistemas nacionales de investigación, regulados desde la administración, han ido otorgando mayor consideración a las revistas como instrumento de difusión investigadora (Castillo-Esparcia \& Carretón, 2010; Ruiz-Pérez et al., 2010). Ese movimiento institucional ha sido seguido, con mayor o menor celeridad, por los investigadores por propio convencimiento o por la necesidad de la mejora económica ya 
sea para acceder a categorías superiores de investigador (Buela-Casal, 2010; Velasco et al., 2012). Todo este cambio en el sistema de evaluación de la actividad investigadora y en los medios de transmisión de las investigaciones suponen un trasvase de estudios a aquellas revistas que se encuentran catalogadas como de calidad. Sin embargo, en los últimos años, la comunidad académica de la Comunicación ha tendido a valorar en exceso la publicación de artículos en revistas indexadas en la base de datos Social Science Citation Index (SSCI), comercializada por Thompson Reuters e implementada desde el Institute for Scientific Information (ISI). También se da una consideración relevante a las revistas recogidas en la base Scopus, a cargo de la editorial Elsevier y accesible, como la anterior, por suscripción. Sin embargo, el peso asignado a estas bases de datos, y en especial al SSCl, ha sido calificado de "abrumador" (Masip, 2011, p. 206) y criticado, entre otros factores, por su falta de transparencia y su sesgo geográfico y lingüístico a favor de la comunidad anglosajona (Archambault et al., 2006), además de profundizar en la dependencia científica del campo de la Comunicación español, europeo o latinoamericano con respecto a las reglas de un mercado marcado por empresas estadounidenses (Reig, 2014).

En el ámbito de las Ciencias Sociales, los principales países en la producción científica son del ámbito anglosajón (Estados Unidos, Reino Unido y Australia), además de China. Por lo que respecta a los países de habla hispánica, solo nos encontramos a España en la séptima posición. Como se puede observar en la Tabla 1, el dato de las citaciones es particularmente negativo, ya que es el tercer país que posee un menor número de citas por artículo, con el dato 0,46 citaciones por documento; por debajo se encuentran India $(0,34)$ y Rusia $(0,21)$.

Tabla 1 Ranking mundial por países en producción de artículos en Ciencias Sociales en el Scimago Journal \& Country Rank (2019).

Fuente. https://www.scimagojr.com/

$\begin{array}{lcccccc}\text { País } & \text { Documentos } & \begin{array}{c}\text { Documentos } \\ \text { citables }\end{array} & \text { Citas } & \text { Autocitas } & \begin{array}{c}\text { Citas por } \\ \text { documento }\end{array} & \text { Índice H } \\ \text { Estados Unidos } & 78.720 & 72.904 & 40.833 & 23.021 & 0,52 & 742 \\ \text { Reino Unido } & 30.270 & 27.687 & 19.481 & 7.655 & 0,64 & 445 \\ \text { China } & 22.140 & 21.420 & 12.430 & 7.637 & 0,56 & 201 \\ \text { Australia } & 15.156 & 14.123 & 10.078 & 3.673 & 0,66 & 296 \\ \text { Alemania } & 14.064 & 13.158 & 8.951 & 3.367 & 0,64 & 305 \\ \text { Canadá } & 12.724 & 11.815 & 7.313 & 2.156 & 0,57 & 364 \\ \text { España } & 12.606 & 12.097 & 5.838 & 2.342 & 0,46 & 208 \\ \text { Italia } & 9.666 & 9.006 & 5.888 & 2.508 & 0,61 & 224 \\ \text { Federación Rusa } & 8.960 & 8.470 & 1.918 & 1.376 & 0,21 & 99 \\ \text { India } & 8.589 & 8.021 & 2.879 & 1.086 & 0,34 & 141\end{array}$


Si atendemos a la Comunicación y al listado de países, sufre algunas modificaciones cuando se observa que España pasa a ocupar la tercera de las posiciones y Brasil ocupa el octavo lugar. Son los dos únicos países iberoamericanos representados en este listado de los 10 primeros (Tabla 2). Por lo que en esta categoría, las posiciones de estos países latinoamericanos mejoran de manera ostensible, convirtiéndose en dos de los mayores productores de artículos científicos.

Tabla 2 Ranking mundial por países en producción de artículos en Comunicación en el Scimago Journal \& Country Rank (2019).

Fuente. https://www.scimagojr.com/

$\begin{array}{lcccccc}\text { País } & \text { Documentos } & \begin{array}{c}\text { Documentos } \\ \text { citables }\end{array} & \text { Citas } & \text { Autocitas } & \begin{array}{c}\text { Citas por } \\ \text { documento }\end{array} & \text { Índice H } \\ \text { Estados Unidos } & 4.798 & 4.563 & 2.340 & 1.366 & 0,49 & 261 \\ \text { Reino Unido } & 1.328 & 1.205 & 682 & 209 & 0,51 & 142 \\ \text { España } & 900 & 880 & 236 & 123 & 0,26 & 73 \\ \text { China } & 865 & 850 & 273 & 130 & 0,32 & 50 \\ \text { Alemania } & 728 & 690 & 422 & 175 & 0,58 & 88 \\ \text { Australia } & 696 & 647 & 348 & 135 & 0,50 & 92 \\ \text { Canadá } & 582 & 534 & 366 & 90 & 0,63 & 89 \\ \text { Brasil } & 464 & 441 & 56 & 26 & 0,12 & 28 \\ \text { Países Bajos } & 403 & 379 & 328 & 99 & 0,81 & 102 \\ \text { Italia } & 376 & 350 & 135 & 53 & 0,36 & 59\end{array}$

Estos datos muestran el potencial de publicación de los investigadores latinoamericanos en el ámbito de la Comunicación. Sin embargo, la alta producción científica no se corresponde con la repercusión del trabajo científico debido a que las citaciones que poseen los textos publicados por los investigadores latinoamericanos son muy bajas. Así, vemos que los datos nos muestran que no se llega de media a una cita por artículo publicado (Tabla 3).

Tabla 3 Citaciones medias de los artículos publicados de acuerdo con el Scimago Journal \& Country Rank (2019).

Fuente. https://www.scimagojr.com/

$\begin{array}{lcc}\text { País } & \text { Documentos } & \text { Citas por documento } \\ \text { España } & 900 & 0,26 \\ \text { Brasil } & 464 & 0,12 \\ \text { Portugal } & 182 & 0,34 \\ \text { Ecuador } & 37 & 0,03 \\ \text { Colombia } & 78 & 0,17\end{array}$




$\begin{array}{lcc}\text { Mexico } & 100 & 0,22 \\ \text { Chile } & 94 & 0,46 \\ \text { Argentina } & 104 & 0,13 \\ \text { Peru } & 16 & 0,44 \\ \text { Cuba } & 11 & 0 \\ \text { Uruguay } & 10 & 0,20 \\ \text { Costa Rica } & 9 & 0,11\end{array}$

Por lo que debemos considerar relevante analizar qué factores hacen que los textos publicados tengan tan escasa repercusión (en citas) por parte de otros investigadores.

\section{La calidad en las revistas científicas. Del factor de impacto a otros índices de calidad}

En la actualidad, para establecer criterios de calidad de las revistas científicas coexisten numerosas iniciativas que están estructuradas en el denominado factor de impacto (FI) y otros criterios bibliométricos.

En el ámbito de las revistas, el factor de impacto se ha convertido, no sin creciente controversia, en el eje central eliminando otros criterios más cualitativos de valoración de los textos científicos. En el ámbito de las revistas científicas el factor de impacto se concreta en dos sistemas de indización de revistas como son el que realiza Web of Science (WoS) y Scopus. En el caso de WoS nos encontramos que todas las revistas incluidas en el índice están catalogadas en dos grandes grupos como son el SCIE (Science Citatios Index Espanded, que reúne a las revistas de los ámbitos de Ciencias) y el SSCI (Social Science Citation Index, que reúne a las revistas de Ciencias Sociales). Las revistas de Comunicación se encuentran incluidas en el SSCl. El factor de impacto que genera WoS se denomina Journal Citation Reports (JCR).

Por lo que respecta a Scopus, su factor de impacto se denomina SCImago Journal Ranking (SJR).

El cálculo del factor de impacto consiste en la cuantificación entre citas recibidas y números de artículos publicados por una revista a través de la siguiente fórmula:

FI (2019) $=\frac{\text { Citas en } 2019 \text { a documentos publicados en } 2017 \text { y } 2018}{\text { Documentos citables publicados en } 2017 \text { y } 2018}$ 
Al margen del factor de impacto, existen otros índices de valoración de las revistas científicas, como pueden ser Latindex, SciELO o Redalyc (en el contexto de América Latina) y ERIH PLUS (Europeo), entre otros.

En el caso de WoS, posee otros sistemas de catalogación de las revistas que incluye índices ajenos (SciELO, por ejemplo) y otros propios. Existe una confusión entre WoS y JCR, ya que no son lo mismo. En el caso de WoS, actúa como una gran base de datos que nos permite búsquedas de artículos científicos que están ubicados en numerosas bases de datos. A su vez, WoS tiene unos directorios de revistas en los que incluye a determinadas revistas que, para ellos, son relevantes en su campo de conocimiento y que les permiten elaborar el factor de impacto (JCR), al mismo tiempo que crear listados de revistas de importancia en su campo. Para WoS esos tipos de revistas están incluidas en lo que denominan Core Collection (colección principal).

Como vemos, dentro de la Core Collection encontramos catálogos de revistas en dos grandes grupos:

- $\quad$ con factor de impacto que incluye a Science Citation Index Expanded (SCIE) y Social Sciences Citation Index (SSCI);

- $\quad$ otros índices también significativos como son Arts \& Humanities y Emerging Sources Citations Index (ESCl). Estos dos índices tienen, en España, unos criterios de calidad muy relevantes para la evaluación de la actividad científica. Son índices selectivos que realiza WoS sobre aquellas revistas que poseen calidad contrastada.

Según los datos recogidos en 2019, existen 92 revistas incluidas en JCR en el ámbito de la Comunicación. La mayoría son del ámbito anglosajón con una presencia marginal de revistas del contexto latinoamericano. Así, en el caso de las revistas de Comunicación, incluidas en el JCR, solo aparecen dos revistas españolas como son Comunicar (Q1) y El profesional de la Información (Q2).

Las revistas con factor de impacto están divididas en cuartiles a partir del factor de impacto, tal como se pueden ver en las siguientes tablas.

Tabla 4 Revistas del primer cuartil en el ámbito de la comunicación en el JCR (2019). Fuente. https://jcr.clarivate.com/JCRLandingPageAction.action

\section{Ranking}

1

2
Título de la revista

Journal of Advertising

Political Communication
Factor de impacto

6,302

5,912 


\begin{tabular}{|c|c|c|}
\hline 3 & Journal of Computer-Mediated Communication & 5,366 \\
\hline 4 & Communication Methods and Measures & 5,281 \\
\hline 5 & Journal of Communication & 4,846 \\
\hline 6 & New Media \& Society & 4,577 \\
\hline 7 & Information Communication \& Society & 4,559 \\
\hline 8 & Digital Journalism & 4,476 \\
\hline 9 & Communication Monographs & 3,843 \\
\hline 10 & Communication Research & 3,758 \\
\hline 11 & International Journal of Advertising & 3,606 \\
\hline 12 & Human Communication Research & 3,540 \\
\hline 13 & Comunicar & 3,375 \\
\hline 14 & Journalism & 3,179 \\
\hline 15 & Social Media + Society & 2,807 \\
\hline 16 & Policy And Internet & 2,763 \\
\hline 17 & International Journal of Press-Politics & 2,612 \\
\hline 18 & Mobile Media \& Communication & 2,545 \\
\hline 19 & European Journal of Communication & 2,500 \\
\hline 20 & Public Opinion Quarterly & 2,494 \\
\hline 21 & Media Psychology & 2,397 \\
\hline 22 & Information Society & 2,378 \\
\hline 23 & Journal of Social And Personal RE & 2 , \\
\hline
\end{tabular}

Tabla 5 Revistas del segundo cuartil en el ámbito de la comunicación en el JCR (2019). Fuente. https://jcr.clarivate.com/JCRLandingPageAction.action

\section{Ranking}

24

25

26

27

28

29

30

\section{Título de la revista}

Journalism Studies

Public Understanding of Science

Science Communication

Public Relations Review

Telecommunications Policy

Journal of Advertising Research

Journalism \& Mass Communication Quarterly

\section{Factor de impacto}

2,345

2,338

2,328

2,321

2.224

2,169

2,121 
31

32

33

34

35

36

37

38

39

40

41

42

43

44

45

46
Media Culture \& Society

Health Communication

Communication Theory

Journal of Public Relations Research

International Communication Gazette

International Journal of Conflict Management

Mass Communication and Society

Environmental Communication-A Journal of Natu-

$$
\text { re and Culture }
$$

International Journal of Public Opinion Research

Journal of Information Technology \& Politics

Written Communication

Convergence-The International Journal of Re-

search Into New Media Technologies

Critical Studies In Media Communication

Journal of Broadcasting \& Electronic Media

Feminist Media Studies

Journal of Health Communication
2,000

1,965

1,905

1,897

1,877

1,806

1,792

1,787

1,771

1,727

1,714

1,616

1,616

1,607

1,596

Tabla 6 Revistas del tercer cuartil en el ámbito de la comunicación en el JCR (2019). Fuente. https://jcr.clarivate.com/JCRLandingPageAction.action

\section{Ranking}

47

48

49

50

51

52

53

54

55

56

57

\section{Título de la revista}

Research on Language and Social Interaction

Profesional de la Información

Journalism Practice

Personal Relationships

Discourse Studies

Discourse \& Communication

Management Communication Quarterly

Media and Communication

Discourse \& Society

Games and Culture

Discourse Context \& Media
Factor de impacto

1,591

1,580

1,542

1,522

1,483

1,475

1,453

1,400

1,390

1,388

1,380 
Communications-European Journal of Communication Research

Tabla 7 Revistas del cuarto cuartil en el ámbito de la comunicación en el JCR (2019). Fuente. https://jcr.clarivate.com/JCRLandingPageAction.action

\section{Ranking}

70

71

72

\section{Título de la revista}

International Journal of Communication

Quarterly Journal of Speech

Journal of Language and Social Psychology

Social Semiotics

Communication and Critical-Cultural Studies

Javnost-The Public

leee Transactions on Professional Communication

Interaction Studies

Rhetoric Society Quarterly

Journal of Applied Communication Research

Argumentation

Journal of Media Ethics

Television \& New Media

Narrative Inquiry

\section{Factor de impacto}

1,194

1,182

1,162

1,160

1,058

1,024

0,980

0,980

0,961

0,959

0,904

0,867

0,835

0,811 
84

85

86

87

88

89

90

91

92
Asian Journal of Communication

Media International Australia

Translator

Visual Communication

African Journalism Studies

Continuum-Journal of Media \& Cultural Studies

Text \& Talk

Journal of African Media Studies

Tijdschrift voor Communicatiewetenschap
0,743

0,616

0,565

0,523

0,420

0,376

0,328

0,245

0,083

Por lo que respecta a la evolución de las revistas en SJR, nos encontramos que la primera revista latinoamericana es Interface: Comunicação, Saúde, Educação (Brasil) que aparece indexada en 2004. Posteriormente entran en el sistema El Profesional de la Información (España), en 2006, y Comunicar (España), en 2008.

En la actualidad, existen 16 revistas latinoamericanas, con una presencia mayoritaria de publicaciones de Brasil (nueve). En el caso de las revistas latinoamericanas que se encuentran en SJR, aparecen 16 con una preponderancia en los niveles bajos de los cuartiles (tres y cuarto), siendo solo tres de ellas incluidas en el Q2 (Cuadernos. Info - Chile -, Comunicación y Sociedad - México - e Interface: Comunicação, Saúde, Educação - Brasil).

Tabla 8 Revistas latinoamericanas en SJR (2019).

Fuente. https://www.scimagojr.com/

\begin{tabular}{|c|c|c|c|c|c|c|c|c|c|}
\hline $\begin{array}{l}\text { Título } \\
\text { (pais) }\end{array}$ & SJR & $\begin{array}{l}\text { Îndi- } \\
\text { ce } \\
\text { H }\end{array}$ & $\begin{array}{l}\text { Total de } \\
\text { docu- } \\
\text { mentos } \\
\text { (2019) }\end{array}$ & $\begin{array}{c}\text { Total } \\
\text { docu- } \\
\text { mentos } \\
\text { (3 años) }\end{array}$ & $\begin{array}{c}\text { Total } \\
\text { de refe- } \\
\text { rências } \\
\text { (2019) }\end{array}$ & $\begin{array}{c}\text { Total } \\
\text { de } \\
\text { citas (3 } \\
\text { años) }\end{array}$ & $\begin{array}{l}\text { Docu- } \\
\text { mentos } \\
\text { citables } \\
\text { (3 años) }\end{array}$ & $\begin{array}{c}\text { Citas/ } \\
\text { doc. } \\
(2 \\
\text { años) }\end{array}$ & $\begin{array}{c}\text { Árbitro/ } \\
\text { doc. } \\
(2019)\end{array}$ \\
\hline $\begin{array}{l}\text { Cuadernos.info } \\
\text { (Chile) }\end{array}$ & $\begin{array}{c}0,366 \\
\text { Q2 }\end{array}$ & 8 & 29 & 86 & 1.596 & 61 & 81 & 0,68 & 55,03 \\
\hline $\begin{array}{l}\text { Comunicacion } \\
\text { y Sociedad } \\
\text { (México) }\end{array}$ & $\begin{array}{c}0,355 \\
\text { Q2 }\end{array}$ & 5 & 29 & 99 & 1.148 & 39 & 88 & 0,52 & 39,59 \\
\hline
\end{tabular}




\begin{tabular}{|c|c|c|c|c|c|c|c|c|c|}
\hline $\begin{array}{l}\text { Interface: } \\
\text { Comunicacao, } \\
\text { Saude, Edu- } \\
\text { cacao } \\
\text { (Brasil) }\end{array}$ & $\begin{array}{c}0,354 \\
\text { Q2 }\end{array}$ & 19 & 161 & 393 & 4.441 & 183 & 338 & 0,45 & 27,58 \\
\hline $\begin{array}{l}\text { Palabra Clave } \\
\text { (Colombia) }\end{array}$ & $\begin{array}{c}0,265 \\
\text { Q3 }\end{array}$ & 8 & 41 & 124 & 1.548 & 62 & 121 & 0,50 & 37,76 \\
\hline $\begin{array}{l}\text { Informacao e } \\
\text { Sociedade } \\
\text { (Brasil) }\end{array}$ & $\begin{array}{c}0,256 \\
\text { Q3 }\end{array}$ & 5 & 26 & 175 & 745 & 36 & 164 & 0,20 & 28,65 \\
\hline $\begin{array}{l}\text { Texto Livre } \\
\text { (Brasil) }\end{array}$ & $\begin{array}{c}0,198 \\
\text { Q3 }\end{array}$ & 2 & 24 & 87 & 542 & 11 & 81 & 0,14 & 22,58 \\
\hline $\begin{array}{l}\text { Transinfor- } \\
\text { macao } \\
\text { (Brasil) }\end{array}$ & $\begin{array}{c}0,196 \\
\text { Q3 }\end{array}$ & 7 & 29 & 89 & 781 & 37 & 86 & 0,30 & 26,93 \\
\hline $\begin{array}{l}\text { Brazilian } \\
\text { Journalism } \\
\text { Research } \\
\text { (Brasil) }\end{array}$ & $\begin{array}{c}0,192 \\
\text { Q3 }\end{array}$ & 1 & 29 & 43 & 907 & 8 & 39 & 0,21 & 31,28 \\
\hline $\begin{array}{l}\text { Signo y Pensa- } \\
\text { miento } \\
\text { (Colombia) }\end{array}$ & $\begin{array}{c}0,187 \\
\text { Q3 }\end{array}$ & 3 & 20 & 41 & 723 & 20 & 41 & 0,44 & 36,15 \\
\hline $\begin{array}{l}\text { Perspectivas } \\
\text { em Ciencia da } \\
\text { Informacao } \\
\text { (Brasil) }\end{array}$ & $\begin{array}{c}0,178 \\
\text { Q3 }\end{array}$ & 9 & 55 & 148 & 1.728 & 29 & 146 & 0,11 & 31,42 \\
\hline $\begin{array}{l}\text { Revista de Co- } \\
\text { municacion } \\
\text { (Peru) }\end{array}$ & $\begin{array}{c}0,108 \\
\text { Q4 }\end{array}$ & 1 & 31 & 26 & 1.509 & 5 & 25 & 0,20 & 48,68 \\
\hline $\begin{array}{l}\text { Cogency } \\
\text { (Chile) }\end{array}$ & $\begin{array}{c}0,101 \\
\text { Q4 }\end{array}$ & 1 & 4 & 19 & 177 & 0 & 19 & 0,00 & 44,25 \\
\hline $\begin{array}{l}\text { Estudios de } \\
\text { Teoria Literaria } \\
\text { (Argentina) }\end{array}$ & $\begin{array}{c}0,101 \\
\text { Q4 }\end{array}$ & 1 & 62 & 48 & 1.469 & 2 & 40 & 0,05 & 23,69 \\
\hline $\begin{array}{l}\text { Comunicacao } \\
\text { Midia e Con- } \\
\text { sumo } \\
\text { (Brasil) }\end{array}$ & $\begin{array}{c}0,100 \\
Q 4\end{array}$ & 1 & 28 & 57 & 599 & 2 & 51 & 0,04 & 21,39 \\
\hline
\end{tabular}




\begin{tabular}{|c|c|c|c|c|c|c|c|c|c|}
\hline $\begin{array}{l}\text { Discursos Foto- } \\
\text { graficos }\end{array}$ & 0,100 & 2 & 0 & 80 & 0 & 0 & 74 & 0,00 & 0,00 \\
\hline $\begin{array}{l}\text { Caracol } \\
\text { (Brasil) }\end{array}$ & & 0 & 22 & 0 & 352 & 0 & 0 & 0,00 & 16,00 \\
\hline
\end{tabular}

En el caso de las revistas españolas, encontramos a 17, siendo la mayoría ubicada también en los cuartiles bajos (tres y cuatro). Solo aparece una revista en el Q1 (Comunicar) y tres en el Q2 (Profesional de la información, Revista Latina de Comunicación Social y Comunicación y Sociedad).

Tabla 9 Revistas españolas en SJR (2019).

Fuente. https://www.scimagojr.com/

\begin{tabular}{|c|c|c|c|c|c|c|c|c|c|}
\hline Título & SJR & $\begin{array}{c}\text { Îndice } \\
\mathbf{H}\end{array}$ & $\begin{array}{l}\text { Total de } \\
\text { docu- } \\
\text { mentos } \\
\text { (2019) }\end{array}$ & $\begin{array}{l}\text { Total } \\
\text { docu- } \\
\text { mentos } \\
\text { (3 años) }\end{array}$ & $\begin{array}{l}\text { Total } \\
\text { de refe- } \\
\text { rências } \\
\text { (2019) }\end{array}$ & $\begin{array}{l}\text { Total } \\
\text { de ci- } \\
\text { tas (3 } \\
\text { años) }\end{array}$ & $\begin{array}{l}\text { Docu- } \\
\text { mentos } \\
\text { citables } \\
\text { (3 años) }\end{array}$ & $\begin{array}{l}\text { Citas/ } \\
\text { doc. } \\
(2 \\
\text { años) }\end{array}$ & $\begin{array}{c}\text { Árbitro/ } \\
\text { doc. } \\
\text { (2019) }\end{array}$ \\
\hline $\begin{array}{l}\text { Review of } \\
\text { Commu- } \\
\text { nication } \\
\text { Research }\end{array}$ & $\begin{array}{c}1,116 \\
\text { Q1 }\end{array}$ & 4 & 4 & 5 & 570 & 20 & 5 & 4,00 & 142,50 \\
\hline Comunicar & $\begin{array}{c}1,092 \\
\text { Q1 }\end{array}$ & 32 & 40 & 120 & 1655 & 432 & 120 & 3,14 & 41,38 \\
\hline $\begin{array}{l}\text { Profesional } \\
\text { de la Infor- } \\
\text { macion }\end{array}$ & $\begin{array}{c}0,480 \\
\text { Q2 }\end{array}$ & 25 & 100 & 331 & 5.189 & 414 & 331 & 1,19 & 51,89 \\
\hline $\begin{array}{l}\text { Revista La- } \\
\text { tina de Co- } \\
\text { municacion } \\
\text { Social }\end{array}$ & $\begin{array}{c}0,423 \\
\text { Q2 }\end{array}$ & 14 & 55 & 238 & 2.196 & 199 & 238 & 0,89 & 39,93 \\
\hline $\begin{array}{l}\text { Comuni- } \\
\text { cacion y } \\
\text { Sociedad }\end{array}$ & $\begin{array}{c}0,331 \\
\text { Q2 }\end{array}$ & 13 & 60 & 156 & 2.622 & 117 & 152 & 0,69 & 43,70 \\
\hline Educar & $\begin{array}{c}0,283 \\
\text { Q2 }\end{array}$ & 2 & 29 & 26 & 1.127 & 11 & 24 & 0,46 & 38,86 \\
\hline $\begin{array}{l}\text { Estudios } \\
\text { Sobre el } \\
\text { Mensaje Pe- } \\
\text { riodistico }\end{array}$ & $\begin{array}{c}0,195 \\
\text { Q3 }\end{array}$ & 12 & 75 & 269 & 2.432 & 56 & 264 & 0,20 & 32,43 \\
\hline
\end{tabular}




\begin{tabular}{|c|c|c|c|c|c|c|c|c|c|}
\hline Signa & $\begin{array}{c}0,182 \\
\text { Q3 }\end{array}$ & 4 & 46 & 157 & 1.604 & 17 & 152 & 0,13 & 34,87 \\
\hline Analisi & $\begin{array}{c}0,171 \\
\text { Q3 }\end{array}$ & 3 & 12 & 31 & 506 & 12 & 31 & 0,39 & 42,17 \\
\hline Scire & $\begin{array}{c}0,159 \\
\text { Q3 }\end{array}$ & 4 & 12 & 45 & 426 & 9 & 45 & 0,27 & 35,50 \\
\hline $\begin{array}{l}\text { Historia y } \\
\text { Comunica- } \\
\text { cion Social }\end{array}$ & $\begin{array}{c}0,156 \\
\text { Q3 }\end{array}$ & 7 & 42 & 98 & 1.524 & 20 & 92 & 0,20 & 36,29 \\
\hline Ibersid & $\begin{array}{c}0,146 \\
\text { Q3 }\end{array}$ & 2 & 13 & 56 & 241 & 14 & 56 & 0,24 & 18,54 \\
\hline $\begin{array}{l}\text { Cultura, } \\
\text { Lenguaje y } \\
\text { Representa- } \\
\text { cion }\end{array}$ & $\begin{array}{c}0,139 \\
\text { Q3 }\end{array}$ & 3 & 18 & 62 & 708 & 14 & 61 & 0,16 & 39,33 \\
\hline $\begin{array}{l}\text { IC Revista } \\
\text { Cientifica de } \\
\text { Informacion } \\
\text { y Comunica- } \\
\text { cion }\end{array}$ & $\begin{array}{c}0,116 \\
\text { Q4 }\end{array}$ & 1 & 0 & 34 & 0 & 5 & 34 & 0,21 & 0.00 \\
\hline Tripodos & $\begin{array}{c}0,115 \\
\text { Q4 }\end{array}$ & 1 & 11 & 20 & 357 & 5 & 20 & 0,25 & 32,45 \\
\hline Loquens & $\begin{array}{c}0,114 \\
\text { Q4 }\end{array}$ & 1 & 4 & 16 & 193 & 1 & 16 & 0,06 & 48,25 \\
\hline Brumal & $\begin{array}{c}0,100 \\
Q 4\end{array}$ & 1 & 25 & 70 & 657 & 3 & 69 & 0,04 & 26,28 \\
\hline
\end{tabular}

Finalmente, Portugal tiene incluidas en 2019 a seis revistas en SJR. Una de ellas incluida en el Q1 (Media and Communication), y cuatro en los cuartiles bajos (tercero y quarto). 
Tabla 10 Revistas de Portugal incluidas en SJR (2019).

Fuente. https://www.scimagojr.com/

\begin{tabular}{|c|c|c|c|c|c|c|c|c|c|}
\hline Título & SJR & $\begin{array}{l}\text { Îndi- } \\
\text { ce H }\end{array}$ & $\begin{array}{c}\text { Total de } \\
\text { docu- } \\
\text { mentos } \\
\text { (2019) }\end{array}$ & $\begin{array}{l}\text { Total } \\
\text { docu- } \\
\text { mentos } \\
\text { (3 años) }\end{array}$ & $\begin{array}{l}\text { Total } \\
\text { de refe- } \\
\text { rências } \\
\text { (2019) }\end{array}$ & $\begin{array}{c}\text { Total } \\
\text { de } \\
\text { citas (3 } \\
\text { años) }\end{array}$ & $\begin{array}{l}\text { Docu- } \\
\text { mentos } \\
\text { citables } \\
\text { (3 años) }\end{array}$ & $\begin{array}{l}\text { Citas/ } \\
\text { doc. (2 } \\
\text { años) }\end{array}$ & $\begin{array}{c}\text { Árbitro/ } \\
\text { doc. } \\
\text { (2019) }\end{array}$ \\
\hline $\begin{array}{l}\text { Media and } \\
\text { Communi- } \\
\text { cation }\end{array}$ & $\begin{array}{c}0,618 \\
\text { Q1 }\end{array}$ & 15 & 97 & 150 & 4.617 & 299 & 121 & 1,96 & 47,60 \\
\hline $\begin{array}{l}\text { Observa- } \\
\text { torio }\end{array}$ & $\begin{array}{c}0,193 \\
\text { Q3 }\end{array}$ & 9 & 20 & 161 & 1.037 & 42 & 160 & 0,29 & 51,85 \\
\hline $\begin{array}{l}\text { Media and } \\
\text { Jornalismo }\end{array}$ & $\begin{array}{c}0,140 \\
\text { Q3 }\end{array}$ & 1 & 40 & 31 & 1.034 & 5 & 31 & 0,16 & 25,85 \\
\hline $\begin{array}{l}\text { Comunica- } \\
\text { çao e Socie- } \\
\text { dade }\end{array}$ & $\begin{array}{c}0,123 \\
\text { Q4 }\end{array}$ & 1 & 28 & 52 & 1.171 & 5 & 50 & 0,10 & 41,82 \\
\hline $\begin{array}{l}\text { Estudos em } \\
\text { Comuniça- } \\
\text { cao }\end{array}$ & $\begin{array}{r}0,112 \\
\text { Q4 }\end{array}$ & 2 & 14 & 104 & 511 & 6 & 101 & 0,06 & 36,50 \\
\hline Cinema & & 0 & 9 & 0 & 471 & 0 & 0 & 0,00 & 52,33 \\
\hline
\end{tabular}

En el caso de Scopus, las revistas anglosajonas (Gran Bretaña y Estados Unidos) suponen el $67,35 \%$ del total de revistas en Comunicación. Y ese porcentaje se incrementa si consideramos las revistas incluidas en el Q1 ya que suponen el $86,36 \%$.

Tabla 11 Países de procedencia de revistas de Comunicación en Scopus. Fuente. https://www.scimagojr.com/

$\begin{array}{lccccc}\text { Países } & \mathbf{N}^{\circ} \text { de revistas/porcentaje } & \mathbf{Q 1} & \mathbf{Q 2} & \mathbf{Q 3} & \text { Q4 } \\ \text { Reino Unido } & 102(39,84 \%) & 18 & 32 & 28 & 24 \\ \text { Estados Unidos } & 73(28,51 \%) & 39 & 7 & 11 & 16 \\ \text { Países Bajos } & 19(7,42 \%) & 4 & 11 & 2 & 2 \\ \text { Alemania } & 11(4,29 \%) & 2 & 4 & 3 & 2 \\ \text { España } & 9(3,51 \%) & 2 & 3 & 3 & 1 \\ \text { Brasil } & 5(1,95 \%) & 0 & 1 & 3 & 1 \\ \text { Francia } & 4(1,56 \%) & 0 & 1 & 1 & 2 \\ \text { Austria } & 2 & 1 & 1 & 0 & 0\end{array}$




\begin{tabular}{l} 
Portugal \\
Suecia \\
Suiza \\
Croacia \\
India \\
Italia \\
Australia \\
Malasia \\
Lituania \\
Finlandia \\
Dinamarca \\
Corea del Sur \\
Chile \\
Nueva Zelanda \\
México \\
Canadá \\
Emiratos Árabes \\
Unidos \\
\hline
\end{tabular}

2

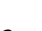

2

\section{2}

2

2

2

2

1

1

1

1

1

Unidos

Otro de los elementos esenciales de las revistas científicas es la necesidad de que el acceso a la información sea público. En este sentido, las revistas de acceso abierto son escasas, ya que, de las 399 revistas recogidas en 2019 de Comunicación, en el sistema SJR, solo 83 de ellas $(20,8 \%)$ son de acceso abierto. Entre las revistas de acceso abierto se encuentran todas las latinoamericanas, con la excepción de Caracol. Sin duda, esa situación supone un esfuerzo a la democratización del conocimiento que aportan las revistas de Comunicación.

Tabla 12 Revistas iberoamericanas en Scopus Fuente. https://www.scimagojr.com/

$\begin{array}{lccc}\text { Título de la revista } & \text { Cuartil (SJR) } & \text { Factor de impacto } & \text { País } \\ \text { Comunicar } & \mathrm{Q} 1 & 0,851 & \text { España } \\ \text { Profesional de la Información } & \mathrm{Q} 2 & 0,601 & \text { España } \\ \text { Revista Latina de Comunicación Social } & \mathrm{Q} 2 & 0,509 & \text { España } \\ \text { Media and Communication } & \mathrm{Q} 2 & 0,444 & \text { Portugal }\end{array}$


Interface: Comunicação, Saúde, Educação

Cuadernos.info

Comunicación y Sociedad (España)

Comunicacion y Sociedad (México)

Palabra Clave

Estudios Sobre el Mensaje Periodístico

Historia y Comunicación Social

Transinformação

Signa

Observatorio

Informação e Sociedade

Perspectivas em Ciência da Informação

Estudos em Comunicação

Signo y Pensamiento

Cultura, Lenguaje y Representación

Review of Communication Research

Scire

IC Revista Científica de Información y Comunicación

Analisi

Ibersid

Comunicação e Sociedade

Comunicação Mídia e Consumo

Texto Livre

Brumal

Discursos Fotográficos

Brazilian Journalism Research

Revista de Comunicación

Loquens
Colombia

España

España

Brasil

España

Portugal

Brasil

Brasil

Portugal

Colombia

España

España

España

España

España

España

Portugal

Brasil

Brasil

España

Q4

0,1

Brasil

Brasil

Perú

España

\section{Críticas al factor de impacto}

Los dos sistemas que están basados en el factor de impacto han venido recibiendo una serie de críticas como son: 
1. sólo incluyen revistas y dejan de lado a otros materiales;

2. metodología para calcular el factor de impacto y la incorrecta interpretación;

3. errores en el vaciado de datos con la identificación de los autores;

4. desigual cobertura geográfica e idiomática;

5. el impacto de una revista no es el de todos sus artículos: $50 \%$ de citas al $15 \%$ de artículos;

6. no distinguen las que se hacen a artículos de investigación de las de contribuciones menores;

7. favorecen áreas muy desarrolladas o con gran número de investigadores;

8. el factor de impacto no es una medida directa de la calidad sino de la frecuencia con que se citan los artículos en un año.

En el ámbito internacional de la investigación han surgido diversas iniciativas que limitan el valor de las métricas como medida de la calidad de la investigación científica. Es el caso de la Declaración de San Francisco de Evaluación de la Investigación (2012), que se produjo en una reunión de un grupo de editores de revistas académicas durante la reunión anual de la American Society for Cell Biology (ASCB), en San Francisco, California, el 16 de diciembre de 2012. En esta declaración se plantea la necesidad de que los índices de impacto no sean los únicos ni principales elementos de valoración de calidad en la investigación. Existen limitaciones a la utilización de las métricas como son:

1. las distribuciones de citas dentro de las revistas son muy sesgadas;

2. las propiedades del factor de impacto son específicas de cada campo: es un compuesto de múltiples tipos de artículos altamente diversos, incluyendo trabajos de investigación primaria y revisiones;

3. los factores de impacto pueden ser manipulados (o evaluados) por la política editorial;

4. y los datos utilizados para calcular el factor de impacto no son transparentes ni están abiertamente disponibles para el público. 
Asimismo, en el denominado Manifiesto Leiden (Hicks et al., 2015) se resalta la necesidad de la evaluación de la ciencia sea contextualizada y aplicada por los objetivos perseguidos por los contextos nacionales y por índices de calidad. Para ello, los precursores de ese manifiesto planean una serie de principios, como son:

1. la evaluación cuantitativa tiene que apoyar la valoración cualitativa por expertos;

2. el desempeño debe ser medido de acuerdo con las misiones de investigación de la institución, grupo o investigador;

3. La excelencia en investigación de relevancia local debe ser protegida;

4. Los procesos de recopilación y análisis de datos deben ser abiertos, transparentes y simples;

5. Los datos y análisis deben estar abiertos a verificación por los evaluados;

6. las diferencias en las prácticas de publicación y citación entre campos científicos deben tenerse en cuenta;

7. la evaluación individual de investigadores debe basarse en la valoración cualitativa de su portafolio de investigación;

8. debe evitarse la concreción improcedente y la falsa precisión;

9. deben reconocerse los efectos sistémicos de la evaluación y los indicadores;

10. los indicadores deben ser examinados y actualizados periódicamente.

\section{Otros índices}

Al margen del papel hegemónico que en algunos países ha adquirido el factor de impacto como elemento de valoración de la calidad de una revista, existen otros sistemas que coexisten y que intentan ampliar los criterios estrictamente cuantitativos. Así tenemos experiencias como Latindex, SciELO o Redalyc, como sistemas del ámbito latinoamericano o experiencias como ERIH PLUS, en el ámbito europeo.

En este sentido, es necesario el fomento de sistemas de evaluación de la calidad científica de las investigaciones propias para los contextos culturales del ámbito 
hispanoamericano que soslayen la hegemonía de modelos que implican poco a nuestras revistas y a nuestros investigadores. De ahí, que sea necesario aumentar el número de revistas algo que ya es un proceso que se viene realizando en las últimas décadas. En la siguiente tabla observamos unos datos que analizan la fecha de creación de las revistas de Comunicación incluidas en Latindex. Así, es claro que, desde el año 2010, existe un creciente proceso de generación de nuevos espacios de difusión de las investigaciones, lo que supone un aire fresco al ecosistema preexistente. Ahora lo que se debe realizar son estrategias de mejoras de los procesos de contenidos y de estructura de las publicaciones para adecuarse a los estándares de toda publicación científica. Para ello, Latindex está desarrollando una magnífica labor con la difusión de las principales características que debe poseer una revista científica, que sirve como listado de chequeo de una publicación ${ }^{1}$.

Tabla 13 Año de creación de las revistas por países.

Fuente. Elaboración propia con datos de los informes proporcionados por Latindex.

$\begin{array}{lcccc}\text { Pais } & \mathbf{2 0 1 0} & \mathbf{2 0 0 0 - 2 0 0 9} & -\mathbf{2 0 0 0} & \begin{array}{c}\text { Número total de } \\ \text { revistas }\end{array} \\ \text { Brasil } & 17,85 \% & 57,14 \% & 25 \% & 56 \\ \text { España } & 37,50 \% & 35,41 \% & 28,08 \% & 48 \\ \text { Chile } & 0 \% & 75 \% & 25 \% & 4 \\ \text { Uruguay } & 33,33 \% & 33,33 \% & 33,33 \% & 3 \\ \text { Costa Rica } & 0 \% & 0 \% & 100 \% & 1 \\ \text { Paraguay } & 100 \% & 0 \% & 0 \% & 1 \\ \text { Colombia } & 0 \% & 33,33 \% & 66,66 \% & 9 \\ \text { Venezuela } & 0 \% & 55,55 \% & 44,44 \% & 9 \\ \text { Portugal } & 11,11 \% & 77,77 \% & 11,11 \% & 9 \\ \text { Argentina } & 27,27 \% & 45,45 \% & 27,27 \% & 11 \\ \text { Ecuador } & 33,33 \% & 33,33 \% & 33,33 \% & 3 \\ \text { México } & 11,11 \% & 33,33 \% & 55,55 \% & 9 \\ \text { Perú } & 33,33 \% & 50 \% & 16,66 \% & 6 \\ \text { Bolivia } & 0 \% & 0 \% & 100 \% & 1\end{array}$

\footnotetext{
${ }^{1}$ Ver https://www.latindex.org/latindex/meto2
} 


\section{Referencias}

Archambault, E., Vignola-Gagné, E., Côté, G., Larivière V., \& Gingras, Y. (2006). Benchmarking scientific output in the social sciences and humanities: The limits of existingdatabases. Scientometrics, 68 (3), 329-342. https://doi.org/10.1007/s11192-006-0115-z

Buela-Casal, G. (2010). Índices de impacto de las revistas científicas e indicadores para medir elrendimiento de los investigadores. Revista de psicodidáctica, 15(1), 3-19. https://www.ehu.eus/ojs/index.php/psicodidactica/article/view/731/606

Castillo-Esparcia, A. (2011). El rol de las publicaciones científicas en Comunicación en el EEES: Indexación e impacto. Revista Internacional de Relaciones Públicas, 1(1), 135-154. https://doi.org/10.5783/RIRP-1-201107-135-154

Castillo-Esparcia, A., \& Carretón, M. C. (2010). Investigación en Comunicación. Estudio bibliométrico de las revistas de Comunicación en España. Comunicación y Sociedad, 23(2), 289-327. https://hdl.handle. net/10171/27872

Declaración de San Francisco de Evaluación de la Investigación, 2012, http://scielo.sld.cu/scielo. php?script=sci_arttext \&pid=S0864-34662014000100017

Hicks, D., Wouters, P., Waltman, L., Rijcke, S., \& Rafols, I. (2015). Bibliometrics: The Leiden Manifesto for research metrics. Nature, 520(7548), 429-431. https://www.nature.com/news/bibliometrics-the-leiden-manifesto-for-research-metrics-1.17351

Masip, P. (2011). Efecto Aneca: Producción española en comunicación en el Social Science Citation Index. Anuario ThinkEPI, 5, 206-210. https://dialnet.unirioja.es/descarga/articulo/3647635.pdf

Reig, R. (2014). La investigación dependiente: Crítica estructural al sistema JCR. Ámbitos, Revista Internacional de Comunicación, 27. https://www.redalyc.org/articulo.oa?id=168/16845649001

Ruiz-Pérez, R., Delgado, E., \& Jiménez-Contreras, E. (2010). Principios y criterios utilizados en España por la Comisión Nacional Evaluadora de la Actividad Investigadora (CNEAI) para la valoración de las publicaciones científicas: 1989-2009. Psicothema, 22(4), 898-908. https://www.redalyc.org/pdf/727/72715515055.pdf

Velasco, B., Bouza, J. M. E., Pinilla, J. M., \& San Román, J. A. (2012). La utilización de los indicadores bibliométricos para evaluar la actividad investigadora. Aula abierta, 40(2), 75-84. https://dialnet.unirioja.es/descarga/ articulo/3920967.pdf 